\title{
Charge transport mechanism of polyaniline/ZnO nanocomposites based on inorganic/organic heterojunctions
}

\author{
Lei Han ${ }^{1, a}$, Wen $\mathrm{Li}^{1}$, Chao Meng ${ }^{1}$, Yan Chen ${ }^{1}$ and Shan Fan ${ }^{1}$ \\ ${ }^{1}$ Department of Mechanics, Wenhua College, No. 8 Wenhua Yuan Road, Wuhan 430074, P.R. China
}

\begin{abstract}
The key to improve the photocurrent is how to promote the generation rate and reduce the recombination rate of the photo-hole pair. In this paper, a p-n heterjunction structure in Polyaniline (PANI)/ZnO nanocomposites was formed via mechanical mixing process. Compared with $\mathrm{ZnO}$, PANI doped $\mathrm{ZnO}$ nanocomposites exhibit obviously enhanced response. It might be attributed to the $\mathrm{p}$-n heterojunctions formed between $\mathrm{p}$-type and n-type enhanced response, enhancing the charge separation and in turn promoting the photoconductivity of the film. The 5at-\%PANI doped $\mathrm{ZnO}$ nanocomposites demonstrates the highest response.
\end{abstract}

\section{Introduction}

Heterojunction composites of conjugated polymers and semiconductor nanoparticles (NPs) have attracted a great deal of attention in photocatalysis [1], solar cells [2], gas sensors [3] and photodiodes [4]. The hybrid organic-inorganic semiconductor junctions are envisaged to complement each others' functional advantages while overcoming their respective drawbacks. The high quality schottky junction or $\mathrm{p}-\mathrm{n}$ junctions formed the composites interface have improved charge separation. [5]

Among the inorganic nanoparticles, $\mathrm{ZnO}$ nanomaterials have received enormous interest in exclusive electrical, catalytic, electronic and optical properties due to their wide band gap (3.3eV), low power threshold sand high exciton binding energy $(\sim 60 \mathrm{meV})$ as well as their low cost. [6] The conducting polymers, which have substantial $\pi$-electrons through the polymeric chain, are the excellent hosts for the trapping of the carriers from the semiconducting nanomaterias.[7] PANI has received much attention as a promising material for microelectronic devices due to due to unique electrical, optical, photoelectric properties and good environmental stability. [8] PANI is extensively used as p-type material which could be deposited by various techniques such as wet, chemical, and electrophoretic methods on n-type semiconducting electrodes for $p$-n heterostructure devices. [9] Various methods have been widely used for the synthesis of the nanocomposites of PANI with the inorganic nanoparticles $\mathrm{ZnO}$.

The results indicate that $\mathrm{ZnO}$ together with PANI, an inorganic/organic hybrid structure shows a promising application in the UV photodetection. [10-11]Under the light illumination, ZnO and PANI produce photo electric-hole pair. The p-n heterointerface between $\mathrm{ZnO}$ and PANI can promote to reduce the recombination rate of the photo-generated electron-hole pair. Although there are many methods to prepare the composite material, the main purpose is to improve the transfer of the photogenerated electrons, which the PANI can act as a bridge to transfer the photo-generated electrons from

\footnotetext{
${ }^{\mathrm{a}}$ Corresponding author : xiaohan0522@163.com
} 
the $\mathrm{ZnO}$. However, the interfacial area in the structure may accelerate recombination, suggesting the need to manage the content of $\mathrm{ZnO}$ or PANI.

In this work, we designed porous $\mathrm{PANI} / \mathrm{ZnO}$ nanocomposites with the different mass ratios of PANI:ZnO from 0:100 to 100:0 to evaluate photoconductivity response. The photoconductivity measurement is made to test the photoelectrical response of each sample, and the best content of PANI is confirmed based on the photoconductivity. The aim of this paper is to evoke the importance of a subject that has been overlooked, as well as to establish a method to calculate the percolation threshold of p-n heterojunction nanocomposites, which is based on photoconductivity measurement. The PANI/ZnO nanocomposites have been characterized by FT-IR, UV visible spectroscopy and SEM.

\section{Experimental Section}

\subsection{Materials}

Commercial PANI (average size $1 \mu \mathrm{m}$ ) and $\mathrm{ZnO}$ (average size $80 \mathrm{~nm}$ ) were used. Other chemicals used in the experiment were of analtytically pure grade. All of them were used as received without any pretreatment. Fixed quantity of $\mathrm{ZnO}$ nanoparticles were mixed with different weight of doped polyaniline powder. Six different ratios of $\mathrm{ZnO}(\mathrm{Z})$ and Polyaniline(P) were studied labeled as ZP100:0(ZP000), ZP99:1(ZP001), ZP95:5(ZP005), ZP90:10(ZP010), ZP50:50(ZP050), and $\mathrm{ZP0}$ :100(ZP100) were prepared with different weight ratio as indicated above.

The two pure powders and a certain amount of organic solvent (a 55:30:10:4:1 weight-\% combination of terpineol, butyl carbitol, di-n-butyl phthalate, span 85, andethyl-cellulose) were mixed in a 7:3 weight ratio. Organic solvent was used mainly as thickening agent and rheological agent for screen printing. The mass of powder in each combination was $10 \mathrm{~g}$. Each sample was processed in a QM-3SP04 ball miller (Nanjing University Instrument Factory, China) for 3 hours at $250 \mathrm{rpm}$ to obtain a paste suitable for screen printing. The paste was printed onto an Au interdigital electrode which was preprinted on an alumina substrate. The spacing of the fingers in the Au interdigitated electrode is $0.4 \mathrm{~mm}$. The dimensions of each sample were $8 \mathrm{~mm}$ by $6 \mathrm{~mm}$ by $10 \mu \mathrm{m}$ thickness. The latter value was controlled by the screen printing machine (WJ-LCD4050CHL Shenzhen Screen-Star Instrument Co. China). These samples were heat-treated for $30 \mathrm{~min}$ at $200{ }^{\circ} \mathrm{C}$ to eliminate the organic solvent. After the above procedures, a thick porous film was obtained in each case. [12]

\subsection{Photoconductivity Measurement}

The photoconductivity of each device in the material library was measured by a homemade photoconductivity measurement platform. All the samples were located in the stainless steel test chamber and tested under different light sources (UV LED $365 \mathrm{~nm}, 36 \mathrm{~W} / \mathrm{m}^{2}$ ) with constant specific bias voltage and ambient air conditions $\left(20^{\circ} \mathrm{C}\right.$ and below $20 \%$ relative humidity) which was controlled by the dry air generator and the mass flow controller.

A typical conductivity-time curve (in this film) is shown in Figure 4. The bias voltage was added at $10 \mathrm{~s}$, and the dark conductivity was obtained. Illumination by LED was initiated at $20 \mathrm{~s}$ and conductivity was monitored, reaching a maximum (at which the generation and recombination of electron-hole pairs achieves dynamic equilibrium) by 160 seconds for all samples. The LED was turned off at $180 \mathrm{~s}$. Finally the bias voltage was unloaded at $350 \mathrm{~s}$. All the photoconductivity measurements were lasted for $360 \mathrm{~s}$.

To obtain the $I-V$ characteristics, current was continuously measured as function of voltage. In the light irradiation experiments, we used UV LED array $\left(365 \mathrm{~nm}, 36 \mathrm{~W} / \mathrm{m}^{2}\right.$ ) and the lamp-to-sample distance was $1 \mathrm{~cm}$. 
The morphologies of the prepared samples were observed on field-emission scanning microscopy (FSEM, Sirion 200 FEI). Room-temperature Fourier transform infrared (FT-IR) spectra were recorded in the range of $4000-500 \mathrm{~cm}^{-1}$ on VERTEX 70 (made by Bruker Co., Germany) using the KBr pellet technique. The UV-vis DRS spectra (Lambda 35, PerkinElmer) was used to obtain the reflectance spectra of the samples over 300-800 nm.

\section{Results and Disscuccion}

\subsection{Characterizations}

The FT-IR spectra of the different composites are showed in Figure 1. The band at $490 \mathrm{~cm}^{-1}$ observed in the spectra of Figure 1 is assigned to $\mathrm{ZnO}$.[13] All the characteristic bands of PANI [14], such as the $\mathrm{C}=\mathrm{C}$ stretching deformation of the quinoid $\left(1556 \mathrm{~cm}^{-1}\right)$ and benzenoid $\left(1472 \mathrm{~cm}^{-1}\right)$ rings, the $\mathrm{C}-\mathrm{N}$ stretch of secondary aromatic amines $\left(1300 \mathrm{~cm}^{-1}\right)$, the aromatic $\mathrm{C}-\mathrm{H}$ in-plane bending $\left(1140 \mathrm{~cm}^{-1}\right)$, and the N-H stretching $\left(3447 \mathrm{~cm}^{-1}\right)$, can be observed in Figure 1.

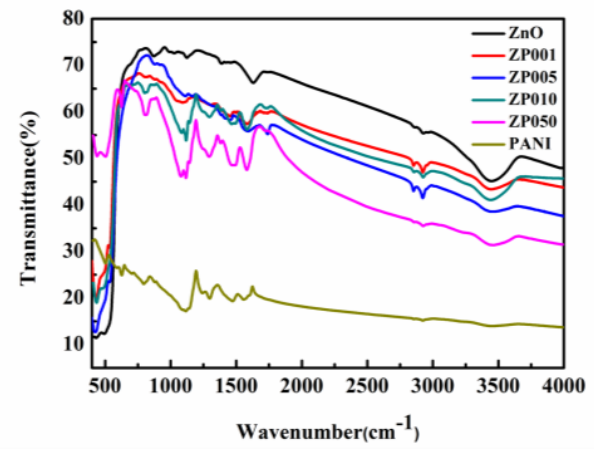

Figure 1 FT-IR spectra of the samples

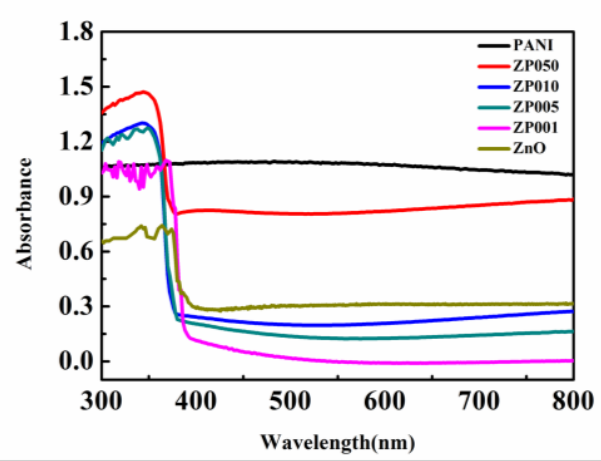

Figure 2 UV-visible adsorption spectra of the samples

Figure 2 shows the UV-visible adsorption spectra of the PANI/ZnO nanocomposites and the PANI. The PANI shows sharp and a broader bands at 350 and $650 \mathrm{~nm}$ which are ascribed to $\pi \rightarrow \pi^{*}$ transitions of the benzenoid groups and $n \rightarrow \pi^{*}$ transitions due to polaron formation into the conducting PANI, respectively.[14] It can be clearly seen that the absorption edge of the ZnO/PANI nanocomposites could be shifted into visible spectrum. This red shift might occur due to the interaction between the hydroxyl groups of $\mathrm{ZnO}$ and the quinoid ring of emeraldine salt (ES) which facilitates the charge transfer from highly reactive imines groups at quinoid unit of $\mathrm{ES}$ to $\mathrm{ZnO}$ nanomaterials via partial hydrogen bonding. [15] Additionally, the relative intensity of the peaks increases with the increased weight percent ratio of PANI nanomaterials in PANI/ZnO nanocomposites. This increased intensities of the peaks might at tribute to the enhanced hydrogen bonding in the form of $\mathrm{NH}-(\mathrm{PANI}) \cdots \mathrm{O}-\mathrm{Zn}[16]$.

The SEM images of the samples are shown in Figure 3. The composite is composed of the $\mathrm{ZnO}$ nanoparticles with the diameter of $40 \sim 60 \mathrm{~nm}$ and the PANI with500 $\mathrm{nm}$. From the SEM image, the size of PANI particles is larger than that of the $\mathrm{ZnO}$ particles. When the content of $\mathrm{ZnO}$ is larger than $50 \%$, the PANI particles are surrounded by $\mathrm{ZnO}$, which like as islands in $\mathrm{ZnO}$. 

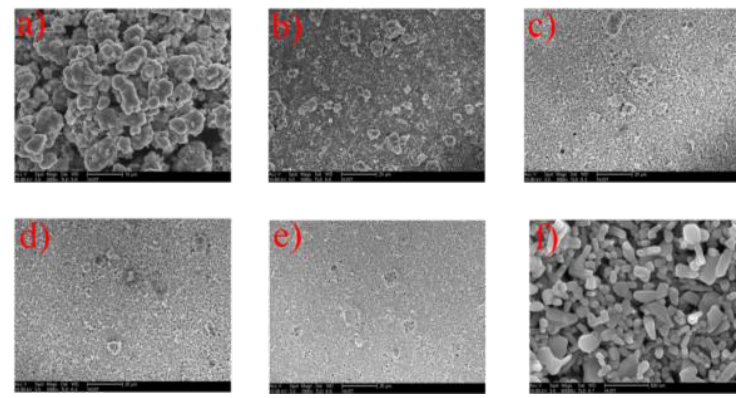

Figure 3 SEM images of the samples

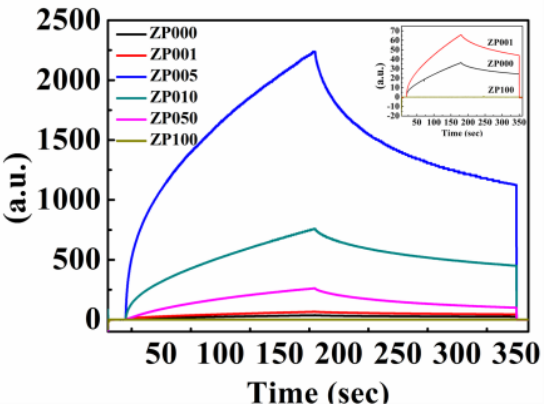

Figure 4 Photoconductivity responses of the samples

\subsection{Photoconductivity response of the PANI/ZnO nanocomposites}

Figure 4 shows the photoconductivity responses of composites with the mass ratios of PANI: $\mathrm{ZnO}$ from 0:100 to 100:0. It is shown that the conducting polymer PANI performs the less response to UV because of the high recombination of electron and hole [16-17]. In the whole experimental range, the $\mathrm{PANI} / \mathrm{ZnO}$ nanocomposites demonstrate much higher response than that of the pure $\mathrm{ZnO}$, indicating that the addition of PANI is beneficial to the UV responsing of the $\mathrm{ZnO}$ nanoparticles. Especially, the 5 at-\%PANI doped $\mathrm{ZnO}$ nanocomposites (ZP005) exhibits markedly higher response than pure, 1, 10, 50 and 100at-\%PANI-ZnO nanocomposites. It also suggests obviously the increasing photoconductivity amplitude can be seen with the increase of the PANI to ZnO from 0/100 to 5/100. When the initial ratios of PANI to ZnO larger than $5 / 100$, the photoconductivity amplitude begins to decrease.

\subsection{I-V characteristics of the PANI/ZnO nanocomposites}

Figure 5 illustrates the $I-V$ characteristics of the n-ZnO/p-PANI (ZP050 and ZP010) nanocomposites heterojunction in the dark and UV irradiation conditions at room temperature, respectively. The linear curve in the Figure 5 (a) can indicate that the ohmic contact is fromed between the $\mathrm{ZnO}$ and PANI (ZP010). The inset on upper side in Figure 5 (a) shows no current flow in the measured voltage range, indicative the resistance of the film being very high. The two non-linear curves in Figure 5 (b) exhibit almost symmetric characteristic with an extended plateau suggestive of tunneling conduction through the PANI/ZnO nanocomposites. This result confirms that a $\mathrm{p}-\mathrm{n}$ junction was successfully formed.

(a)

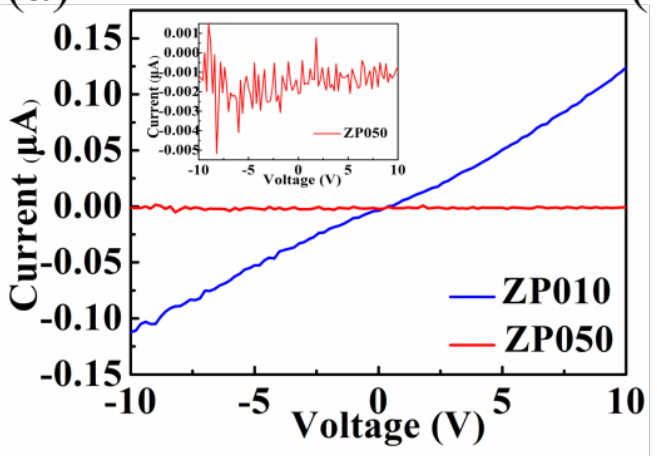

(b)

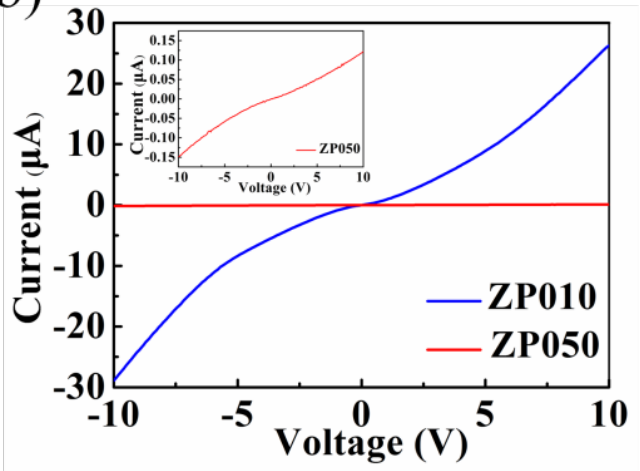

Figure $5 I-V$ characteristics of the PANI/ZnO nanocomposites in the dark (a) and in the light (b)

\subsection{Mechanism analysis}


The conducting polymer PANI is p-type semiconductor and can form the holes-accumulation layer in the grain boundary. [14] The metal oxide $\mathrm{ZnO}$ is typical n-type semiconductor and the $\mathrm{ZnO}$ surface can easily adsorb oxygen molecules. [18-19] The chemical adsorb oxygen molecules, which trap the free electrons from the conduction band of $\mathrm{ZnO}$, can transform to negative oxygen ions. [20] So the electrons-depletion layer is formed at the grain boundary of $\mathrm{ZnO}$. Based on the research of Lin et al. [10], both of the energy bands will bend up nearby the surface of the $p-n$ heterjunction (as shown in Figure $6(\mathrm{a}))$.

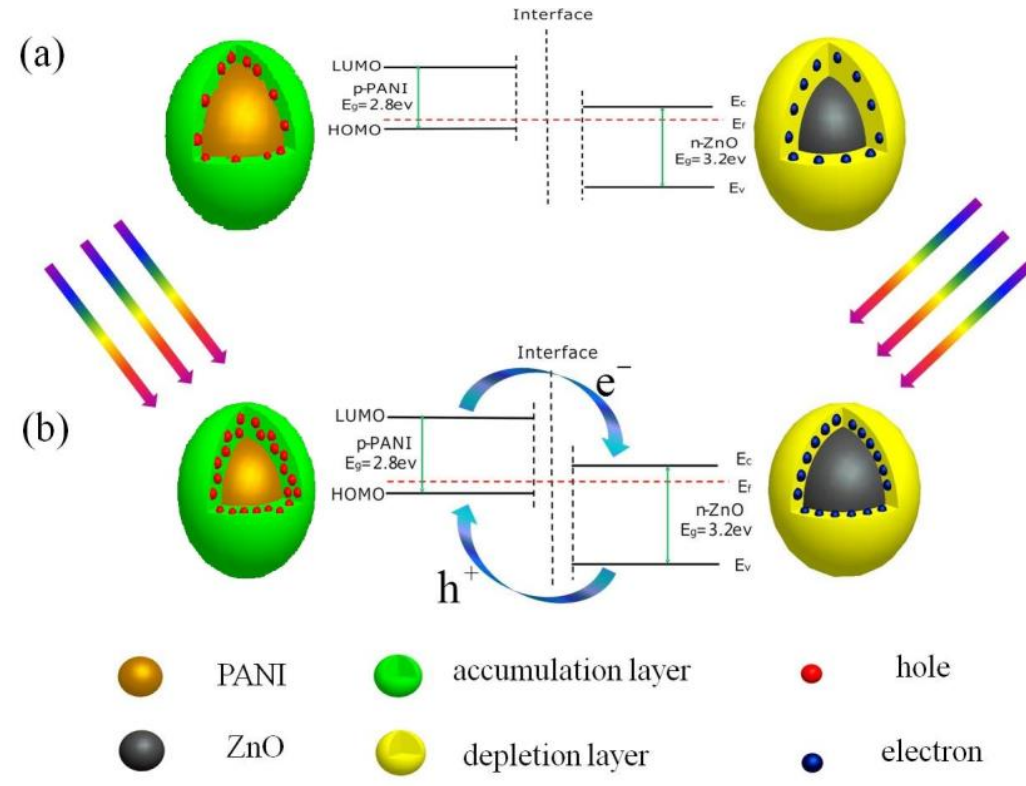

Figure 6 Schematic illustration of the energy bands for the grain boundary of the PANI/ZnO nanocomposites (a) in the dark (b) in the light

Under UV illumination, the interface of $\mathrm{ZnO}$ and PANI will be changed by photo-generated electrons and holes. The photo-generated holes migrate to the $\mathrm{ZnO}$ surface and react with the negative oxygen ions. The photo-generated electrons transfer between the LUMO of PANI and the CB of ZnO. The photo-generated holes transfer between the VB of $\mathrm{ZnO}$ and the HOMO of PANI. These lead to the decrease of holes-accumulation layer of PANI and the increase of the electrons-depletion layer of $\mathrm{ZnO}$, which is as shown in Figure 6 (b).

Here $\mathrm{e}^{-}$and $\mathrm{h}^{+}$represent photogenerated electrons and holes, respectively. The subscript $\mathrm{Z}$ and $\mathrm{P}$ mean the related subjects stay in $\mathrm{ZnO}$ and PANI, respectively. During the on and off illumination test, several processes might have happened:

$$
\begin{gathered}
\mathrm{ZnO}+\operatorname{photo}(U V) \rightarrow e_{(Z)}^{-}+h_{(Z)}^{+} \\
P A N I+\operatorname{photo}(U V) \rightarrow e_{(P)}^{-}+h_{(P)}^{+} \\
e_{(P)}^{-} \rightarrow e_{(Z)}^{-} \\
h_{(Z)}^{+} \rightarrow h_{(P)}^{+} \\
e_{(Z)}^{-}+O_{2} \rightarrow O_{2}^{-} \\
O_{2}^{-}+h_{(Z)}^{+} \rightarrow O_{2} \uparrow \\
e_{(Z)}^{-}+h_{(Z)}^{+} \rightarrow \text { recombination(heat) } \\
e_{(P)}^{-}+h_{(P)}^{+} \rightarrow \text { recombination(heat) }
\end{gathered}
$$


During illumination, $\mathrm{O}_{2}{ }^{-}$reacts with photo induced holes. [20] The adsorbed oxygen molecules are released with the help of this reaction, which will lower the barrier height as proposed by Muraoka.[20]

\section{Conclusion}

A facile, simple and environment friendly mechanical mixing process method was used to synthesise PANI doped $\mathrm{ZnO}$ p-n heterojunction nanocomposites. Compared with pure $\mathrm{ZnO}$ nanoparticles, PANI doped $\mathrm{ZnO}$ p-n heterojunction samples demonstrate enhanced UV responding performances.It might be ascribed to the formation of $\mathrm{p}-\mathrm{n}$ heterojunctions between $\mathrm{p}$-type PANI and n-type $\mathrm{ZnO}$ interfaces, further promoting the generation rate and reducing the recombination rate of the photo-generated electron-hole pair. Among all fabricated samples, the 5at-\% PANI doped $\mathrm{ZnO}$ nanocomposites demonstrate the highest responses in the UV light. All experiments indicate the 5at-\% PANI doped $\mathrm{ZnO}$ p-n heterojunction nanocomposites as a promising sensing candidate for UV detection in practical application. The PANI is like some islands that storage the holes from the VB of the $\mathrm{ZnO}$. This island-structure is helpful to separate electron-hole pairs.

\section{Reference}

1. Q.L. Li, C.R. Zhang, J.Q. Li, Appl. Surf. Sci. 257, 944-948 (2010).

2. A.A. Khan, M. Khalid, J. Appl. Polym. Sci. 117, 1601-1607 (2010).

3. J. Huang, T.L. Yang, Y.F. Yang, Y. Wang, S. Wang, J. Nat. Gas Chem. 20, 515-519 (2011).

4. O. Game, U. Singh, T.Y. Kumari, A. Banpurkar, S. Ogale, Nanoscale 6, 503-513 (2014).

5. W.W. Wu, S. Bai, N.Y. Cui, F. Ma, Z.Y. Wei, Y. Qin, E. Xie, Sci. Adv. Mater. 2, $402-406$ (2010).

6. L. Irimpan, V.P. N. Nampoori, P. Radhakrishnan, Sci. Adv. Mater. 2, 578-582 (2010).

7. Z.H. Mbhele, M.G. Salemane, C.G.C.E. Sittert, J.M. Nedeljkovic, V. Djokovic, A.S. Luyt, Chem. Mater. 15, 5019-5024 (2003).

8. S. Ameen, M.S. Akhtar, Y.S. Kim, O.B. Yang, H.S. Shin, Microchim. Acta 172,471-478 (2011).

9. F. Ahmed, S. Kumar, N. Arshi, M.S. Anwar, S.Y. Lee, G.S. Kil, D.W. Park, B.H. Koo, C.G. Lee, Thin Solid Films 519, 8375-8378 (2011).

10. Y.J. Lin, T.H. Su, J.C. Lin, Y.C. Su, Synthetic Met. 162, 406-409 (2012).

11. J. Wu, H.Y. Li, Y. Liu, C.S. Xie, J. Appl. Phys. 110, 123513-123516 (2011).

12. Z.J. Zou, Y. Liu, H.Y. Li, Y.C. Liao, C.S. Xie, J. Comb. Chem. 12, 363-369 (2010).

13. T. Guo, L.S. Wang, D.G. Evans, W. S. Yang, J. Phys. Chem. C 114, 4765-4772 (2010).

14. S. Ameen, M.S. Akhtar, Y.S. Kim, O.B. Yang, H.S. Shin, Colloid \& Polym. Sci. 289, 415-421 (2011).

15. D.X. Han, Y. Chu, L.K. Yang, Y. Liu, Z.X. Lv, Colloid. \& Surf. A: Physicochemical \& Eng. Asp. 259, 179-187 (2005).

16. Q.W. Tang, L. Lin, X. Zhao, K. Huang, J.H. Wu, Langmuir 28, 3972-3978 (2012).

17. Y.M. Xiao, G.Y. Han, Y.Z. Chang, H.H. Zhou, M.Y. Li, Y.P. Li, J. Power Sources 267, 1-8 (2012).

18. C.S. Lao, M.C. Park, Q. Kuang, Y.L. Deng, A.K. Sood, D. L. Polla, Z. L. Wang, J. Am. Chem. Soc.129, 12096-12097 (2007).

19. Y.Q. Bie, Z.M. Liao, P.W. Wang, Y.B. Zhou, X.B. Han,Y. Ye, Q. Zhao, X.S. Wu, L. Dai, J. Xu, Adv. Mater. 22, 4284-4287 (2010).

20. Y. Muraoka, N. Takubo, Z. Hiroi, J. Appl. Phys. 105, 103702-103707(2009). 